\title{
Scale problems in assessment of hydrogeological parameters of groundwater flow models
}

\author{
Marek Nawalany*, Grzegorz Sinicyn \\ Faculty of Environmental Engineering, Warsaw University of Technology, Nowowiejska 20, 00-653 Warszawa, Poland \\ *corresponding author, e-mail: marek.nawalany@is.pw.edu.pl
}

\begin{abstract}
An overview is presented of scale problems in groundwater flow, with emphasis on upscaling of hydraulic conductivity, being a brief summary of the conventional upscaling approach with some attention paid to recently emerged approaches. The focus is on essential aspects which may be an advantage in comparison to the occasionally extremely extensive summaries presented in the literature. In the present paper the concept of scale is introduced as an indispensable part of system analysis applied to hydrogeology. The concept is illustrated with a simple hydrogeological system for which definitions of four major ingredients of scale are presented: (i) spatial extent and geometry of hydrogeological system, (ii) spatial continuity and granularity of both natural and man-made objects within the system, (iii) duration of the system and (iv) continuity / granularity of natural and man-related variables of groundwater flow system. Scales used in hydrogeology are categorised into five classes: micro-scale - scale of pores, meso-scale - scale of laboratory sample, macro-scale - scale of typical blocks in numerical models of groundwater flow, local-scale - scale of an aquifer/aquitard and regional-scale - scale of series of aquifers and aquitards. Variables, parameters and groundwater flow equations for the three lowest scales, i.e., pore-scale, sample-scale and (numerical) block-scale, are discussed in detail, with the aim to justify physically deterministic procedures of upscaling from finer to coarser scales (stochastic issues of upscaling are not discussed here). Since the procedure of transition from sample-scale to block-scale is physically well based, it is a good candidate for upscaling block-scale models to local-scale models and likewise for upscaling local-scale models to regional-scale models. Also the latest results in downscaling from block-scale to sample scale are briefly referred to.
\end{abstract}

Keywords: scale problem, upscaling, hydraulic conductivity, hydrogeological models

\section{Introduction}

The concept of scale arises at the very first stage of system analysis. Irrespective of whether the system itself is abstract or physical, its analysis always requires that some axiomatic assumptions be made as to its space-time extent and its continuity/granularity. By defining the scale of the system, a scientist encapsulates his or her own experience, expertise or intuition concerning the given class of systems in a few primary concepts which can be understood by other people, or at least by some selected group of people. When 'the goal-seeking' definition of the system (Klir, 1969) is used, the space-time extent and continuity/granularity of the system's objects are purposely defined, their attributes and the relationships between the attributes so that some problem can be solved or some predefined goal achieved. The system approach and scale definition are especially useful when the system under consideration is complex, e.g., when it contains a large number of physical objects having a spectrum of 'sizes' and when relationships between these objects are a mixture of discrete and continuous interactions. By assuming 'proper' scale and structure of the system the 'seek solution' to the problem may be 'hit', whereas by taking the 'wrong' scale the system's behaviour and departure from the goal may not be explained. The issue is how to distinguish between 'proper' and 'wrong' scale attribut- 
ed to the problem. There is one familiar answer to this question - setting a scale for a given system or problem depends on prior knowledge, experience and intuition of the system. Usually, experience from the past is used when setting a scale for 'typical' systems. When confronted with 'new type' systems, however, an intellectual leap forward must be made; setting a scale for such cases is a kind of 'art'. At the present time, when information accessibility concerning any given discipline (and hence 'typical systems' of the discipline) is not restricted, 'the experience' can be understood as 'global knowledge'. Still, for problems or systems of 'new type' invention of new concepts in the systems' scale definition may become necessary.

Definitely, hydrogeological systems are complex Earth systems that require system approach and scale definition each time when theoretical issues of groundwater flow or practical problems of groundwater resources are to be solved. The system approach to dynamic hydrogeological systems has been applied by many researchers and extensively presented in the literature. One of the first works was a monograph by Nawalany (1984) as well as the TNO Report (Nawalany, 1987). A chapter in which system analysis was devoted to hydrogeological systems can be found in the book by Zijl \& Nawalany (1993), 'Natural Groundwater Flow'. Subsequently, Zijl also published a book (1993) and a paper (1999) on scale issues in hydrogeology. In this brief treatise, the system approach and scale definition are discussed as aspects of groundwater flow modelling and as part of procedures used for the assessment of parameters in hydrogeological models.

\section{System aspects of groundwater flow modelling}

When considering groundwater flow in porous or fissure rocks in the context of solving practical problems or answering theoretical questions, a number of axiomatic assumptions concerning scale and structure of the hydrogeological system is made in the hope that the physical-mathematical model based on these assumptions will allow to obtain the seek solutions or provide answers to the questions. Aspects of system analysis and scale concepts, chosen here for the sake of illustration, correspond to a simple case of groundwater flow in a single aquifer recharged with water from infiltration, seepage through aquitards or far-distant inflow from adjacent aquifers. There is also a gal- lery of wells which abstract groundwater from the aquifer with the aim of supplying a community with a specified volume of water within the period of one year. The question is whether the given volume of groundwater can be abstracted from the aquifer safely, i.e., without overexploitation of the aquifer's water resources. By considering this goal as guidance, an analysis of the hydrogeological system commences from defining scale features of the groundwater flow model. Typically, the following features of the model that represents a given system are defined as follows:

- spatial extent and geometry of the hydrogeological system. For instance, horizontal and vertical dimensions of an aquifer are specified together with the geometry of aquitards and other aquifers that set (geometrical) limits to a given aquifer.

- spatial continuity and granularity of natural and man-made objects within the systems. By attributing the concept of hydraulic conductivity to the given aquifer, it is specified whether the parameter may have discontinuities (and where) and how hydraulic conductivity does change in space (granularity). As to man-made objects, localisation and abstraction rate of wells in the gallery need to be specified.

- time extent of the system. In this case a one-year period defines the duration of the system operation. It applies to all variables involved - infiltration, seepage through aquitards, far-distant inflow from adjacent aquifers and water abstraction by wells.

- continuity/granularity of natural and man-related variables of the system. Specific discharge and piezometric head are assumed to be natural variables continuous in time and space, whereas abstraction rates in wells may be discontinuous in time (e.g., wells can be switched off for maintenance). By modelling time changes of specific discharge and piezometric head within the aquifer (system), the question if the water supplies from the gallery of wells at the given rate may lead to overexploitation of water resources of the aquifer, may be answered.

Typically, one of the five scale ranges can be considered when resolving hydrogeological issues through modelling groundwater flow. Depending of the formulation of the problem/question, hydrogeologists may set their groundwater flow models within one of the following scales:

- micro-scale - scale of pores (in the order of $10^{-6}$ $10^{-2} \mathrm{~m}$ )

- meso-scale - scale of laboratory sample (in the order of $10^{-2}-10^{0} \mathrm{~m}$ ) 
- macro-scale - scale of typical blocks in numerical models of groundwater flow (in the order of $10^{\circ}-$ $10^{2} \mathrm{~m}$ )

- local-scale - scale of an aquifer (in the order of $10^{2}-10^{3} \mathrm{~m}$ )

- regional-scale - scale of series of aquifers/aquitards (in the order of $10^{3}-10^{6} \mathrm{~m}$ ).

It should be noted that Bear (1979) did use the term macro-scale for the laboratory sample scale (here named meso-scale).

A basic question attributed to each scale is what physical variables are suitable to describe groundwater flow in the subsoil at a given scale? And the question which immediately follows is what type of physical-mathematical equations and what parameters are suitable to describe relationships between these variables? There is also another question: how to pass from variables, equations and parameters of one scale to variables, equations and parameters of the next scale? When the next scale is higher, the procedure is called upscaling and, reversely, when the next scale is lower the procedure is called downscaling. The following section describes procedures that allow for upscaling groundwater flow models from micro-scale to meso-scale models and later from meso-scale to macro-scale models. Knowing the transition rules from meso-scale to macro-scale, a similar (not necessarily exactly the same) procedure may be applied in order to make a transition from macro-scale to local-scale and finally from local-scale to regional-scale. Downscaling is not discussed here; only a brief reference is made to the latest results obtained by Trykozko (2010).

\section{Groundwater flow equations at various hydrogeological scales and transitions between them}

In this section groundwater flow equations in the three smallest scale models and corresponding two transition rules between them are described for setting the basis for transitions to higher-scale models.

Physics-based equations of groundwater flow in the pore-scale are the Navier-Stokes equations of hydrodynamics - the continuity equation and the momentum equation:

$$
\begin{gathered}
\frac{\partial \rho}{\partial t}+\nabla \cdot(\rho v)=0 \\
\rho\left[\frac{\partial v}{\partial t}+v \cdot(\nabla \cdot v)\right]=-\nabla p+\rho g+\nabla \cdot \underline{S}(v, \mu, \xi)
\end{gathered}
$$

where:

$v_{x}, v_{y}, v_{z}$ - pore velocity components (m/s),

$p$ - pressure in a pore $(\mathrm{Pa})$,

$\rho$ - water density in a pore $\left(\mathrm{kg} / \mathrm{m}^{3}\right)$,

$\mu, \xi$ - dynamic and volumetric viscosities in the pore space $(\mathrm{kg} / \mathrm{ms})$,

$\underline{S}$ - stress tensor (Pa); for a Newtonian fluid (e.g. water, air) $\underline{S}=\mu\left(\nabla v+(\nabla v)^{T}\right)+\xi(\nabla \cdot v) I$, (I is the unit tensor).

The four equations - (1) and (2), contain seven variables $v_{x}, v, v, p, \rho, \mu, \xi$. Even with the appropriate initial and boundary conditions there are too many variables in equations (1) and (2). Therefore, three constitutive equations representing phenomenological relationships between (some) variables are added:

$$
\begin{aligned}
& \rho=F\left(p, \chi_{0}, \chi_{1}, \ldots\right) \\
& \mu=G\left(p, \chi_{0}, \chi_{1}, \ldots\right) \\
& \xi=H\left(p, \chi_{0}, \chi_{1}, \ldots\right)
\end{aligned}
$$

where:

$\chi_{0}$ - temperature in a pore $\left({ }^{\circ} \mathrm{C}\right)$,

$\chi_{1}, \ldots$ - other variables, e.g. concentration of some chemical species in pore water $\left(\mathrm{kg} / \mathrm{m}^{3}\right)$ in order to equalise the number of unknowns and the number of equations and hence "close" the system of equations.

\subsection{From pore-scale to laboratory sample- scale}

In order to transit from micro-scale (pore-scale) to meso-scale (of laboratory sample-scale) the concept of the Representative Elementary Volume $(R E V)-U_{o^{\prime}}$ introduced by Bear (1979) can be utilized to average physical variable $G_{\alpha}\left(\underline{x}^{\prime}, t\right)$ specified within phase a in all pores contained in $U_{0}$.

For fixed $\underline{x}$ (centre of REV) and fixed moment of time $t$ new variable $\bar{G}_{\alpha}(\underline{x}, t)$ is defined as

$$
\bar{G}_{\alpha}(\underline{x}, t)=\frac{1}{\left|U_{o}\right|} \iiint_{U_{o}(\underline{x})} G_{\alpha}\left(\underline{x}^{\prime}, t\right) d \underline{x^{\prime}}
$$

where: $\left|U_{0}\right|$ - volume of REV.

According to the Continuity Hypothesis the new variable $\bar{G}_{\alpha}(\underline{x}, t)$ - REV-averaged $G_{\alpha}\left(\underline{x}^{\prime}, t\right)$, is at each moment in time continuous in space and does not depend of shape or volume of REV. The REV-averaging removes two major obstacles faced by modellers when using the pore-scale models in real hydrogeological situations - it allows avoidance 
of variables' discontinuities on complex solid-fluid interfaces and the necessity of defining boundary conditions on these interfaces.

By applying the averaging procedure to continuity equation (1) in the micro-scale is obtained

$\frac{1}{\left|U_{o}\right|} \iiint_{U_{o}(\underline{x})} \frac{\partial \rho}{\partial t} d \underline{x^{\prime}}+\frac{1}{\left|U_{o}\right|} \iiint_{U_{o}(\underline{x})} \nabla \cdot(\rho v) d \underline{x^{\prime}}=0$

which, after a number of transformations, turns into the well-known continuity equation for averaged variables in porous medium sample

$$
\frac{\partial(\bar{\rho} \Theta)}{\partial t}+\nabla \cdot(\bar{\rho} \Theta \bar{v})=0
$$

where:

$\Theta$ - volume of water in a rock sample/volume of rock sample, i.e. soil moisture (-),

$\bar{\rho}$ - averaged density of water in $\operatorname{REV}\left(\mathrm{kg} / \mathrm{m}^{3}\right)$,

$\bar{v}$ - averaged velocity of water in $\operatorname{REV}(\mathrm{m} / \mathrm{s})$.

Similarly, by averaging the momentum equation (2) in micro-scale over REV one obtains

$$
\begin{gathered}
\frac{1}{\left|U_{o}\right|} \iiint_{U_{o}(\underline{x})} \rho\left[\frac{\partial v}{\partial t}+v \cdot(\nabla \cdot v)\right] d \underline{x}^{\prime}= \\
=\frac{1}{\left|U_{o}\right|} \iiint_{U_{o}(\underline{x})}[-\nabla p+\rho g+\nabla \cdot \underline{S}(v, \mu, \xi)] d \underline{x}^{\prime}
\end{gathered}
$$

which, after a number of transformations, turns into the general form of the momentum equation for averaged variables in a porous medium sample

$$
\bar{\rho}\left[\frac{\partial \bar{v}}{\partial t}+\bar{v} \cdot(\nabla \cdot \bar{v})\right]=-\nabla \bar{p}+\bar{\rho} g-F
$$

where:

$F$ - total force acting on water per unit volume of rock sample $\left(\mathrm{N} / \mathrm{m}^{3}\right)$,

$\bar{p}$ - averaged pressure in water in $\operatorname{REV}(\mathrm{Pa})$.

For small water velocities (typical for flows in most of porous rocks) inertia effects are negligible and the left-hand side of equation (7) can be assumed zero whereas the total force acting on the water per unit volume of rock sample (sheer force) can be considered proportional to the pore velocity of the water. In such a case equation (7) turns into Darcy's Law (!)

$$
\bar{v}=-\frac{\kappa}{\Theta \mu}(\nabla \bar{p}-\bar{\rho} g)
$$

where $\kappa$ - coefficient of permeability $\left(\mathrm{m}^{2}\right)$.
Hence, physics-based equations of groundwater flow in a laboratory sample-scale (or meso-scale) are represented by The Continuity Law (5) and the Darcy's Law (8). In their standard form the two equations are expressed (for saturated flow and constant density of water) with the use of specific discharge $q$ and piezometric head $\Phi$

$$
\begin{gathered}
\frac{\partial}{\partial t}(n \bar{\rho})=\nabla \cdot(\bar{\rho} q) \\
q=-k \nabla \Phi
\end{gathered}
$$

Equations (5') and $\left(8^{\prime}\right)$ are combined into the ultimate groundwater flow equation

$$
S_{s} \frac{\partial \Phi}{\partial t}=\nabla \cdot(k \nabla \Phi)
$$

where $S_{s}$ - specific storage $(1 / \mathrm{m})$.

It must be noted that for large water velocities in a porous medium (with Reynolds number about 1) upscaling from the core to the sample scale leads to non-Darcian flow equation. This was demonstrated by Peszyńska \& Trykozko (2013), who used new voxel-based techniques for imaging of real porous media to support pore-to-sample up-scaling. According to its very definition equation (9) is suitable for describing groundwater flow on the sample-scale for small flow velocities. However, the hydrogeological community uses this equation as a basis for numerical modelling of groundwater flow in flow in large hydrogeological systems of porous rocks, irrespective of the sheer fact that, basically, the equation and the systems belong to different scales! This discrepancy, which is overlooked by many modellers, can be (and is) repaired if proper transition is applied.

\subsection{From meso-scale to macro-scale (scale of an aquifer)}

It must be recalled that for the meso-scale typical dimensions of rock samples are in the range of 0.1-1.0 m, whereas in macro-scale models describing groundwater flow in (numerical) model blocks the dimensions are in the order of 1-100 m. Typically, hundreds or thousands of such blocks are combined into one numerical model of an aquifer. Before composing blocks into one integrated model of an aquifer, modellers make an axiomatic assumption that

"in the scale of each modelling block the relation between the gradient of the piezometric head (as a cause) 
and the specific discharge (as an effect) has a form identical with the Darcy Law (8') derived for a sample-scale".

From this assumption it is clear that, in order to use 'the Darcy Law' on the 'block-scale' (macro-scale), block hydraulic coefficient $K_{b}(\underline{\mathbf{x}})$ needs to be found, which at any point in the space of block is consistent with this 'block Darcy Law', i.e. with

$$
(1 / V) \int_{V} q\left(x^{\prime}\right) d x^{\prime}=-K_{b}(x)(1 / V) \int_{V} \nabla \Phi\left(x^{\prime}\right) d x^{\prime}
$$

or, equivalently, with

$$
\langle q(x)\rangle=-K_{b}(x)\langle\nabla \Phi(x)\rangle
$$

where $\langle\varphi\rangle$ is the volume-averaged value of variable $\varphi$ within the block.

The above volume averaging is correct if the grid volumes have rectangular shapes (i.e., the volumes are "real blocks"). However, if the grid volumes have different shapes, the physical volume (in $\mathrm{m}^{3}$ ) has to be replaced with a 'generalised volume' that incorporates the scale factors or the curvilinear co-ordinates. This was clearly demonstrated by Zijl \& Trykozko (2001) for wedge-shaped volumes around a well. However, this is often overlooked by modellers.

Zijl (1993) and Nawalany (1999) showed that, in general, the block hydraulic coefficient $K_{b}(\underline{\mathbf{x}})$ has the following characteristics:

a) $K_{b}(\underline{\mathbf{x}})$ is not an arithmetic average of the hydraulic conductivities (at the sample-scale) in the block

b) $K_{b}(\underline{\mathbf{x}})$ depends of geometry of flow field

c) $K_{b}(\underline{\mathbf{x}})$ is a tensor even if the sample-scale hydraulic conductivity is a scalar variable

d) $K_{b}(\underline{\mathbf{x}})$ is not necessarily a symmetric tensor even if in the sample-scale hydraulic conductivity is a symmetric tensor.

For special cases when

a) flow through the block is uniform

b) hydraulic conductivity tensor is symmetric on the sample-scale,

the following deterministic formulas can be used for calculating corresponding scalar values of block hydraulic conductivity $K_{b}(x)$ provided values of hydraulic conductivity $k\left(x^{\prime}\right)$ at the sample-scale are known:

- for 1-dimensional flow in the $x$ direction parallel to the layers

$$
K_{x}(x)=(1 / V) \int_{V} k\left(x^{\prime}\right) d x^{\prime}
$$

where $V$ is a block volume (one grid block or a cluster of grid blocks),
- for 1-dimensional flow in the $z$ direction normal to the layers

$$
K_{z}(z)=\left\{(1 / V) \int_{V}\left[k\left(z^{\prime}\right)\right]^{-1} d z^{\prime}\right\}^{-1}
$$

where $V$ is a block volume,

- for 2-dimensional flow with $k_{1}$ and $k_{2}$ in a chequerboard pattern

$$
K_{b}=\left(k_{1} k_{2}\right)^{1 / 2}
$$

see Warren and Price (1961) and Trykozko (2007),

- for 2-dimensional flow

$$
K_{b}(x)=\exp \left\{(1 / V) \int_{V} \ln \left(k\left(x^{\prime}\right)\right) d x^{\prime}\right\}
$$

- for 3-dimensional flow with $k_{1}$ and $k_{2}$ in a 'generalised chequerboard' pattern

$$
K_{b}=\left(k_{1} k_{2}\right)^{1 / 2}+\text { correction term }
$$

see Trykozko (2007),

- for 3-dimensional flow

$$
K_{b}(x)=\left\{(1 / V) \int_{V}\left[k\left(x^{\prime}\right)\right]^{1 / 3} d x^{\prime}\right\}^{3}
$$

Formulas (11), (11'), (12') and (13') were derived for special cases a) and b) (above) by Idelman \& Dagan (1993a, b) and Idelman (1993). However, it was shown by Zijl (1993) and Nawalany (1999) that for general case, $K_{b}(\underline{\mathbf{x}})$ depends of solving the flow equation for the block-scale $\left(10^{\prime}\right)$, i.e. it depends of boundary conditions imposed over the block boundaries.

This leads to the idea of imposing two (in 2-D) or three (in 3-D) different sets of boundary conditions ( $i$ $=1,2)$ or $(i=1,2,3)$, respectively, at the block boundaries and calculate (as a solution to the inverse problem) four elements of block hydraulic conductivity tensor $K_{b}(\underline{\mathbf{x}})$ from the corresponding four sets of algebraic equations. The equations representing the block Darcy Law in the $x$ - and $y$-directions for the assumed two sets of boundary conditions $(i=1,2)$,

$$
\begin{aligned}
& \left\langle q_{x}\right\rangle^{i}=K_{b x x}\langle\Delta \Phi / \Delta x\rangle^{i}-K_{b x y}\langle\Delta \Phi / \Delta y\rangle^{i} \\
& \left\langle q_{y}\right\rangle^{i}=K_{b y x}\langle\Delta \Phi / \Delta x\rangle^{i}-K_{b y y}\langle\Delta \Phi / \Delta y\rangle^{i}
\end{aligned}
$$


The method of upscaling from sample-scale to block-scale as outlined above was extended to three-dimensional blocks by Zijl \& Trykozko (1999), Trykozko \& Zijl (2000) and Trykozko (2007). Their numerical experiments confirmed characteristics a) - d) of block tensor $K_{b}(\underline{\mathbf{x}})$.

It must be noted that considerable progress has recently been made by Trykozko et al. $(2008,2009)$ and Trykozko (2010), who founded a new method for numerical upscaling and downscaling (downscaling is the transfer of coarse-scale conductivities to fine-scale conductivities). The idea is based on an inverse model applied in such a way that each grid volume is considered as a zone. Since conventional inverse models such as e.g., PEST (Doherty, 2004), require a prohibitively large number of memory space and computation time, Trykozko et al. (2009) were amongst the first to develop an alternative inversion procedure in which a great many zones, including each grid block as a zone, do not pose any computational problem. Based on this idea, Trykozko (2010) solved the downscaling problem for a number of hypothetical situations (among which the 2-D chequerboard and the 3-D 'generalised checkerboard' pattern. In order to determine the error bounds, that author resolved these problems using both the Conformal-Nodal Finite Element Method (an upper bound method) and the Mixed Hybrid Finite Element Method (a lower bound method). One of the major conclusions from her extensive numerical experiments on different scales used for modelling of groundwater flow is that numerical methods used to estimate the hydraulic conductivity pattern in finer-scale models do converge to the same values of the hydraulic conductivity tensor elements. This means that the proposed procedures are independent of their numerical algorithms.

\section{Summary}

The two lowest scales used in describing groundwater flow in porous rocks differ essentially as to the state variables applicable to each scale. For pore-scale solutions the Navier-Stokes equations describing conservation of mass and momentum determine velocity, pressure and water density within each rock pore, whereas the solution for the sample scale equations (mass balance and Darcy's Law) represent the REV-averaged velocity, pressure and water density (specific discharge and piezometric head in typical hydrogeological terminology) within each rock sample having dimensions of order of $1 \mathrm{~cm}$ to $1 \mathrm{~m}$. Although the pore-scale model has limited applicability to field hydrogeology it is still considered a valuable basis for theoretical research, especially when sorption and chemical reactions are added to the flow model. The sample-scale model, with a tradition of over 100 years, has been successfully used in thousands of applications and verified by properly designed experiments and observations. In the present note the transition from a pore-scale model to a sample-scale model accomplished through REV-averaging provides physical justification for the sample-model and explains to large degree why the model is so successful.

Still there are aspects of groundwater modelling that are in need of more careful explanation. At present, when numerical models of groundwater flow in an aquifer (or in subsoil basins consisting of a number of aquifers) are routinely applied in hydrogeology, extrapolation of sample-scale model parameters to macro-scale parameters has become an issue because it might affect the reliability of the models. The solutions to this problem presented here are based on the assumption that macro-scale groundwater flow equations have essentially the same form as the sample-scale models, i.e., they obey the mass balanced equation and Darcy's Law. As a result, upscaling of hydraulic conductivity from the sample-scale to the volume-scale, often simplified to the block-scale (block as rectangular volume) has to follow a specific procedure as described here. Although some progress has been made recently, an interesting question still remains: whether conventional and recently developed tools for parameter identification (inverse models) are compatible with the volume averaging procedure described in the present paper, or not.

As the procedure of transition from sample-scale to block-scale is physically well based, a similar approach can be applied to upscaling block-scale models to local-scale models and likewise when upscaling local-scale models to regional-scale models.

\section{References}

Bear, J., 1979. Hydraulics of Groundwater. McGraw-Hill, New York, 592 pp.

Doherty, J., 2004. PEST: Model Independent Parameter Estimation. Watermark Numerical Computing, Brisbane, Australia

Idelman, P. \& Dagan, G., 1993a. Upscaling permeability of anisotropic heterogeneous formations 1 . The general framework, Water Resources Research 29, 917-923.

Idelman, P. \& Dagan, G., 1993b. Upscaling permeability of anisotropic heterogeneous formations 2 . General structure and small perturbation analysis, Water Resources Research 29, 925-933. 
Idelman, P., 1993. Upscaling permeability of anisotropic heterogeneous formations 3. Applications, Water Resources Research 29, 935-943.

Klir, G.J., 1969. An Approach to General System Theory. Van Nostrand Reinhold, New York, 323 pp.

Nawalany, M., 1984. Groundwater as dynamical system. Scientific Works of Warsaw University of Technology 86, Warszawa.

Nawalany, M., 1987. FLOSA 3D, Introduction to system analysis of three-dimensional groundwater flow. Report of TNO Institute of Applied Geoscience OS 86-07, Delft.

Nawalany, M., 1999. Scale problem in hydrogeology. Bulletin of Polish Geological Institute 388, 179-190.

Peszyńska, M. \& Trykozko, A., 2013. Pore-to-core simulations of flow with large velocities using continuum models and imaging data. Computational Geoscience 17, 623-645.

Trykozko, A., 2007. Computational aspects of up-scaling in heterogeneous porous media. Workshop: Modeling, Analysis and Simulation of Multiscale Nonlinear Systems, Oregon State University, USA.

Trykozko, A., 2010. Downscaling: fine-scale conductivity identification by inverse modelling. XVIII Conference Computational Modeling in Water Resources, Barcelona, Spain.

Trykozko, A. \& Zijl, W., 2000. Finite Element Methods in numerical up-scaling of 3D permeability distributions. Conference Finite Elements in Flow, Austin, USA.
Trykozko, A., Brouwer, G. \& Zijl, W., 2008. Down-scaling: a complement to homogenization. International Journal of Numerical Analysis and Modeling Suppl. 5, 157-170.

Trykozko, A., Mohammed, G.A. \& Zijl, W., 2009. Down-scaling: the inverse of up-scaling. SIAM Conference on Mathematical and Computational Issues in the Geosciences, Leipzig, Germany.

Warren, J.E. \& Price, H.S. 1961. Flow in Heterogeneous Porous Media. Society of Petroleum Engineers Journal 1, 153-169.

Zijl, W., 1993. Scale Analysis in Groundwater Hydrology. Monograph of Laboratory of Hydrology, Free University Brussels, Series VUB - Hydrologie 24, Brussels

Zijl, W., 1999. Scale aspects of groundwater flow and transport systems. Hydrogeology Journal 7, 139-150.

Zijl, W. \& Nawalany, M., 1993. Natural Groundwater Flow. Lewis Publ. Comp., Boca Raton, London, Tokyo, 315 pp.

Zijl, W. \& Trykozko, A., 1999. From fine-scale to coarse-scale permeability by numerical homogenization and volume averaging. Technical report of TNO, NITG (The Netherlands' Institute of Geosciences Research) 99-4-B, Haarlem.

Zijl, W. \& Trykozko, A., 2001. Numerical Homogenization of the Absolute Permeability Tensor Around a Well. SPE (Society of Petroleum Engineers) Journal 5, 399-408. 